\title{
Direct en-face, speckle-reduced images using angular-compounded Master-Slave optical coherence tomography
}

\author{
René Říha, Manuel J. Marques, Michael R. Hughes, Adrian \\ Bradu, Adrian Podoleanu \\ Applied Optics Group, School of Physical Sciences, University of Kent, Canterbury \\ CT2 7NH, United Kingdom
}

\begin{abstract}
In this paper, an angular compounding method to achieve speckle contrast reduction in optical coherence tomography (OCT) imaging is explored in detail. The angular compounding approach involves collecting multiple images at different angles of incidence, registering the images to correct for induced distortions, and then incoherently summing the images to reduce speckle. The method was experimentally demonstrated with a spectrometer-based Master-Slave enhanced OCT instrument capable of directly generating en-face images. We have investigated the impact of the angular range and number of averaged frames on the degree of speckle artefact reduction, as well as the effect on image resolution and sharpness. The minimum angular step necessary to secure a sufficiently small speckle pattern correlation between the images has also been determined, and the method has subsequently been validated on a biological sample (potato cells).
\end{abstract}


Direct en-face, speckle-reduced images using angular-compounded Master-Slave OCT 2

\section{Introduction}

Optical coherence tomography (OCT) is a non-invasive, non-contact imaging technique based on low-coherence interferometry. It is capable of producing crosssectional representations of structures in real-time with micrometer resolution, and penetration depths of 1 to $2 \mathrm{~mm}$ in scattering tissue. OCT imaging has found numerous applications in biology and medicine [1] as well as in other non-biomedical fields such as non-destructive testing and art conservation [2].

OCT relies on the interference of light back-scattered from the sample with that propagating over a reference optical path. Due to this, OCT requires a certain degree of spatial and temporal coherence from the optical source, and, consequently, coherent detection, which inevitably gives rise to speckle [3]. Speckle phenomena arise when optical paths of different light rays scattered from structures within the sample vary in length on a sub-wavelength scale. The multiple scattered contributions interfere with one another, resulting in granularity of the intensity distribution [4]. Speckle degrades the image contrast of biological tissue [5], obscuring features, and in some cases introducing imaging artefacts which may imply the presence of structures that do not exist [2].

Over the last two decades, a significant number of speckle-reducing methods have been reported in the literature. These can be grouped into four categories $[6,7,5]$, namely those employing polarisation diversity, spatial diversity, frequency diversity, and post-acquisition image processing. Most methods reported in the literature are centred on spatial diversity, with angular compounding (i.e. multiple acquisition of images at different angles of incidence, followed by incoherent summation) being the most frequent implementation $[8,9,10,11,2,12,13,14,15]$. Taking advantage of the interferometric set-up in an OCT system, Liba et al. [16] have employed a moving diffuser plate in the reference arm to remotely vary the speckle pattern, achieving good speckle reduction following incoherent summation. More recently, post-acquisition image processing methods $[17,18,19]$ have become increasingly popular, due to significant improvements in computing power.

While speckle noise affects both B-scan and en-face (C-scan) imaging, most reports on speckle reduction techniques in OCT images have focused on B-scans (cross-section or depth cuts) imagery. This is partly because when employing conventional spectral OCT processing methods, B-scans are produced quicker than en-face images. This is due to the fact that conventional (FFT-based) spectral OCT processing requires the whole 3-D volume of raw data to be FFT-processed prior to obtaining individual en-face cuts, which have to be software-cut from the full processed volume. To generate a speckle-reduced en-face image while employing a technique such as angular compounding, several of these volumes would need to be FFT-processed, followed by en-face image extraction and subsequent average.

Unlike these conventional OCT data processing methods (which may also require kdomain re-linearisation and/or dispersion compensation), Master-Slave OCT performs a comparison operation against pre-recorded/synthesised spectra (called "masks"), with 
Direct en-face, speckle-reduced images using angular-compounded Master-Slave OCT 3

each of these comparison operations yielding a constant depth, en-face OCT image. In other words, it is possible to generate en-face images directly from the acquired raw spectra, which presents significant time savings, especially if a low number of axial points is required [20]. Conventional FT-based OCT methods operate in two steps to deliver an en-face OCT image: firstly, by processing each spectral scan to generate its corresponding A-scan, and assembling the entire data volume; and secondly, by cutting from this data volume the desired en-face slice(s). Hence, by introducing complex Master-Slave OCT processing [21], we can minimise the time required to process the data volume for each angle value when only a few en-face images are required [22]. This should help to reduce the speckle of en-face images by angular compounding more feasible, by reducing the total time required to render the final speckle-reduced en-face image.

In this publication, we perform a rigorous and quantitative study of the effectiveness of the angular compounding method in reducing speckle in en-face images obtained using a spectrometer-based OCT system operating at $840 \mathrm{~nm}$. The method used to vary the angle of incidence in this report is similar to that reported by Hughes et al. [2] and Winetraub et al. [14]; however, in this publication, Complex Master Slave OCT was employed to process the raw data volumes acquired.

The spatial diversity procedure enabled by angle compounding is susceptible to optical aberrations caused by off-axis operation [14], therefore we have also studied the impact of angular compounding on the image sharpness. We also present average speckle size measurements over a range of angles of incidence and depth positions within an homogeneous sample. Finally, in order to reduce the overall time required to obtain speckle-reduced en-face images, we demonstrate the applicability of the complex MasterSlave OCT method $[21,23]$ to produce speckle-reduced en-face images.

\section{Materials and Methods}

\subsection{Speckle quantification}

In previous reports $[4,6,24,25,26]$, the statistical properties of speckle have been analysed, with a key result being that the speckle distribution $p(I)$ for most samples follows a Rayleigh distribution, modelled as

$$
p(I)=\frac{I}{\sigma^{2}} \exp \left(-\frac{I^{2}}{2 \sigma^{2}}\right),
$$

where $\sigma$ is the standard deviation of the distribution, and $p(I)$ is defined for the signal intensity $I \in\langle 0, \infty)$.

In this report, for proper speckle noise reduction assessment, speckle visibility is quantified by the speckle contrast ratio, SCR, which is defined as the standard deviation $\sigma_{A}$ of the intensity in a given uniform area of the image divided by the mean intensity 
Direct en-face, speckle-reduced images using angular-compounded Master-Slave OCT 4

$\langle I\rangle_{A}$ evaluated over the same area,

$$
\mathrm{SCR}=\frac{\sigma_{A}}{\langle I\rangle_{A}}
$$

From equation (2), smaller values of SCR mean lower speckle visibility contrast. In other reports $[24,9,10]$, an equivalent quantity is used, the speckle signal-to-noise ratio, which is defined as the inverse of the SCR. When the speckle is fully modelled by a Rayleigh distribution as presented in Eq. (1), and the illumination beam is assumed to be polarised, the maximum SCR is calculated to be 0.52 .

For $N$ uncorrelated speckle patterns which are then averaged [2], SCR is reduced to $\mathrm{SCR}_{\min }$, given by

$$
\mathrm{SCR}_{\min }=\frac{0.52}{\sqrt{N}}
$$

For the case of angular compounding methods, there can be some degree of residual correlation between frames. For large $N$, this leads to a deviation of the SCR from $\mathrm{SCR}_{\min }$ (Eq. (3)), particularly if a small angular range is used in the compounding procedure. Discussion of the influence of the angular range on the interframe correlation, and hence on the SCR reduction, is deferred to Section 3.

\subsection{SD-OCT experimental setup}

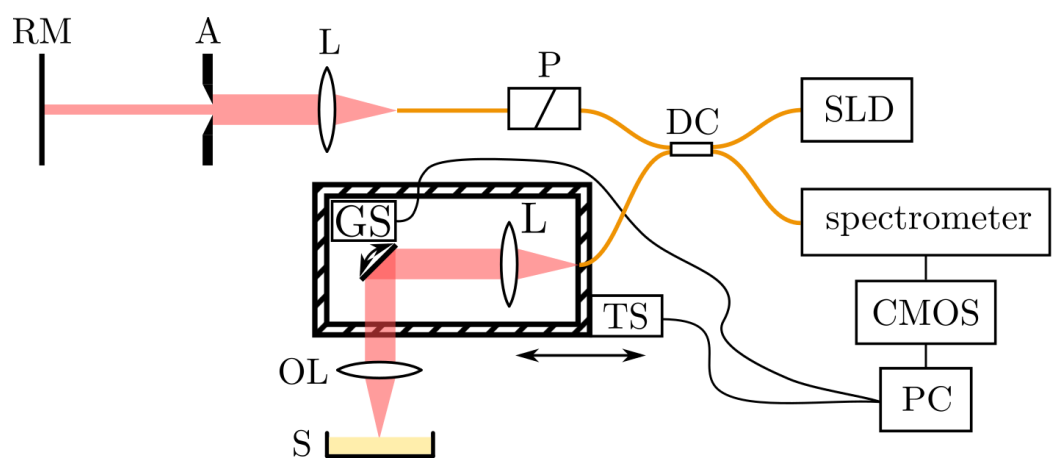

Fig. 1: Schematic of the spectrometer-based OCT set-up for angular compounding. $\mathrm{RM}$ - reference mirror, A - aperture, L - lens, P - fibre polarizer, DC - directional coupler (40\% object arm), TS - linear translation stage, GS - dual-axis galvo scanner head, OL - objective lens $(f=42 \mathrm{~mm}), \mathrm{S}$ - sample, SLD - superluminescent diode $\left(\lambda_{0}=840 \mathrm{~nm}, \Delta \lambda=50 \mathrm{~nm}\right)$.

A custom-built spectrometer-based OCT set-up was assembled, which is shown schematically in Fig. 1. The system uses as an optical source a super-luminescent diode (SLD) of central wavelength $\lambda_{0}=840 \mathrm{~nm}$ and spectral width $\Delta \lambda=50 \mathrm{~nm}$, yielding a theoretical resolution of $6.2 \mu \mathrm{m}$ in air. Light from the source is divided between the sample and reference arms using a 40/60 (40\% for object arm) directional fibre coupler DC. Light reflected from the mirror RM in the reference arm and back-scattered from 
Direct en-face, speckle-reduced images using angular-compounded Master-Slave OCT 5

the sample $\mathrm{S}$ in the object arm are directed by another fibre to the spectrometer, where the resulting spectra are detected by a 2048-pixel CMOS camera (Atmel AVIIVA M2 $\mathrm{CL}$ ) running at an A-line rate of $20 \mathrm{kHz}$. The maximum system sensitivity (measured close to $z=0 \mathrm{~mm}$, and with $0.5 \mathrm{~mW}$ of optical power on the sample) was $85 \mathrm{~dB}$, with $\sim 1.25 \mathrm{~mm}$ axial range at $-6 \mathrm{~dB}$, as shown in Fig. 2 .

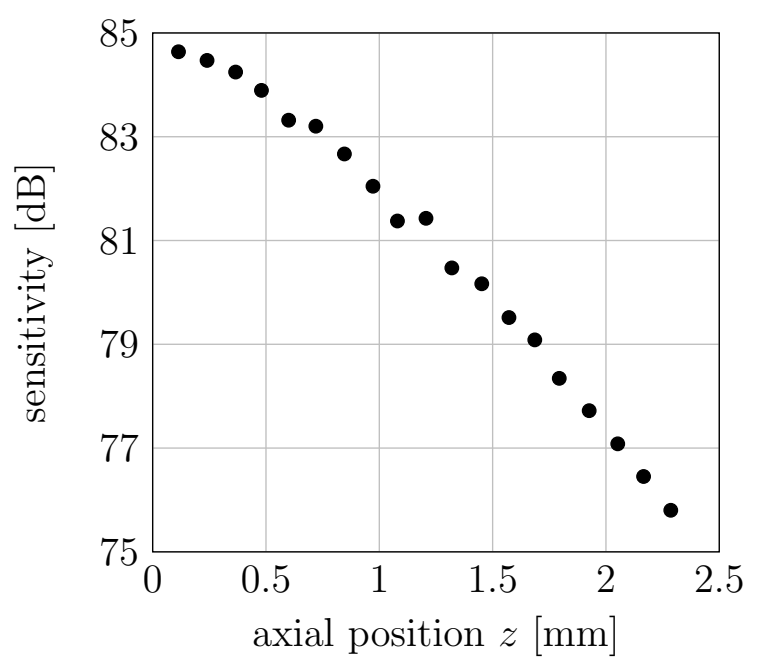

Fig. 2: Sensitivity of the OCT system over axial depth $z$.

The spectra read by the camera are acquired via a CameraLink card (National Instruments PCIe-1427) and further processed in a personal computer (Dell Precision T7500, Intel Xeon X5650 series processor, 48 GB RAM) using the complex Master Slave OCT method $[21,23]$. By performing a comparison operation between oncoming spectra and previously synthesised masks, $200 \times 200$ px en-face images are obtained directly [22] at a frame rate of $0.25 \mathrm{~Hz}$.

In the object arm, the dual-axis galvo scanner head GS (which ensures both $x$ and $y$ lateral scanning) and collimating lens $\mathrm{L}$ are placed on a platform attached to a linear translation stage TS (Newport Z865A), allowing the lateral position of the imaging beam on the objective lens OL (Thorlabs LSM04-BB, EFL $=54 \mathrm{~mm}$, working distance $42 \mathrm{~mm}$ ) to be precisely controlled, and thus the incidence angle on the sample $\mathrm{S}$. The optical power arriving at the sample was measured to be $\approx 0.5 \mathrm{~mW}$; the lateral resolution of the imaging system (at normal incidence) was measured as $10 \mu \mathrm{m}$. Based on a collimated imaging beam of approximately $3 \mathrm{~mm}$, the numerical aperture of the imaging system is 0.028. Assuming a Gaussian beam with an wavelength of $\lambda_{0}=840 \mathrm{~nm}$, we can estimate the theoretical $1 / \mathrm{e}^{2}$ spot size, $w_{0}$ as $\approx 9.6 \mu \mathrm{m}[27]$

$$
w_{0}=\frac{\lambda_{0}}{\pi \theta_{d}}
$$

where $\theta_{d}=\sin \mathrm{NA} \approx \mathrm{NA}$ is the beam divergence. The Raleigh range $z_{R}=\frac{\pi w_{0}^{2}}{\lambda_{0}}$ can 
Direct en-face, speckle-reduced images using angular-compounded Master-Slave OCT 6

then be calculated [27] to quantify the depth of field DoF $=2 z_{R}$ of the system, which has been calculated as DoF $\approx 0.7 \mathrm{~mm}$.

An attempt to measure the lateral resolution at non-normal angles of incidence using a USAF resolution chart printed in a soda lime glass plate was unsuccessful. When tilting the incident angle, rays are scattered by the edges of the printed features in the soda lime glass plate, while specular reflections from the middle of the features are not routed back into the optical system, thus diminishing the overall signal intensity. Due to this, we have only characterised the lateral resolution of the system at normal incidence.

\subsection{Angular compounding}

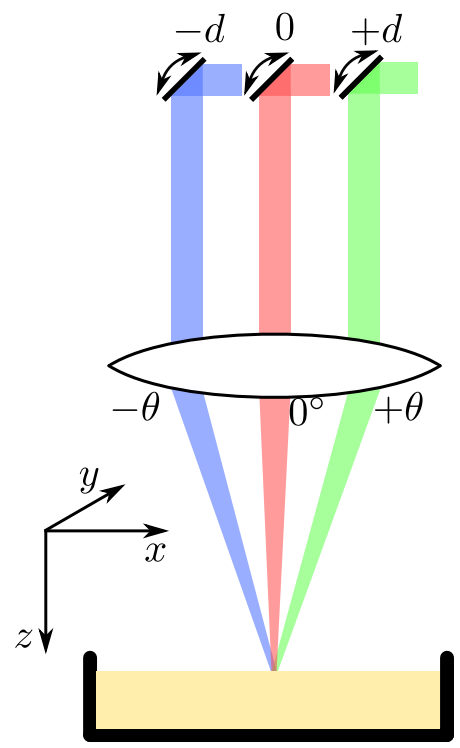

(a)
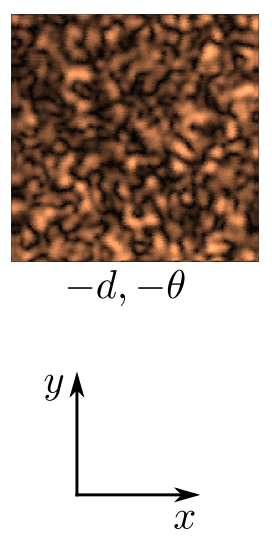

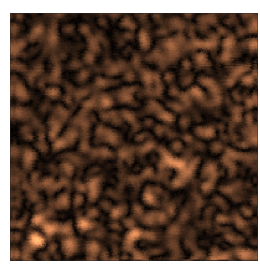

$0,0^{\circ}$

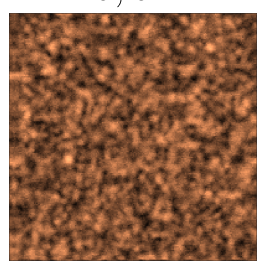

average

$N=3, \Delta \theta=2 \theta$

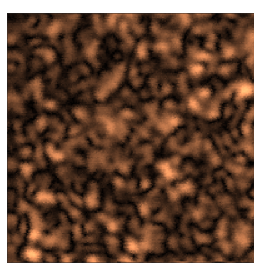

$+d,+\theta$

(b)

Fig. 3: Principle of speckle reduction via angular compounding. (a) Angle of incidence $\theta$ is related to position $d$ of the translation stage TS (Eq. (5)). (b) Illustration of reduction in speckle visibility contrast using three patterns obtained using a sample made of titanium dioxide particles in an epoxy resin (top), and resulting speckle-reduced image (bottom).

Angular compounding speckle reduction methods require the sample to be imaged from multiple angles of incidence, $\theta$, with the resulting frames being incoherently averaged together to yield a speckle-reduced image. If sequentially-acquired frames at the same angle of incidence are incoherently averaged together, no significant decrease in the SCR is observed when compared to a single frame taken at the same depth and location, as the speckle pattern in all images is roughly the same.

As described above, a translation stage TS attached to a platform (hatchet rectangle in Fig. 1) containing both the galvo-scanner head GS and the collimating lens was 
employed. Linear movements of TS causes the beam to become off-axis in relation to the objective lens OL. If the displacement of TS from the case where the imaging beam is aligned with the optical axis is given by $d$, then the beam has an angle of incidence onto the sample of $\theta$ (measured in relation to the normal to the sample), as shown in Fig. 3. Using the small angle approximation, the relation between angle of incidence $\theta$ and $d$ is given simply by

$$
\theta=\arctan \frac{d}{f} \approx \frac{d}{f}
$$

where $f$ is the focal length of the objective lens OL.

The range of angles of incidence $\Delta \theta$, within which $N$ frames are averaged, is given by $\Delta \theta=2 \theta$. The angular step between adjacent frames, $\delta \theta$, is given by

$$
\delta \theta=\frac{\Delta \theta}{N-1} .
$$

We also denote $\theta_{\max }$, as the maximum angle of incidence either side of the normal, $\Delta \theta_{\max }$ as the maximum angular range, and $\delta \theta_{\min }$ as the minimum angular step between subsequent frames.

When the angle of incidence $\theta$ is adjusted (through the movement of TS), the speckle pattern in the image varies. This allows a stack of images to be acquired which captures the same field of view but with speckle patterns that are decorrelated with respect to each other (to some degree). By performing an incoherent average of these frames, some reduction in SCR is achieved, as presented in Section 1. In the next section, attention will be also devoted to the effect of registration on SCR reduction.

Geometrical aberrations (particularly coma) on the imaging beam are expected with increasing angle of incidence $\theta$, with implications on the quality of the en-face images. To assess this (and since we were unable to characterise the lateral resolution of the system at non-normal angles of incidence, as described in the previous section), a 2-D CCD camera (Thorlabs DCC1545M) was placed in the focal plane of the objective lens OL, and the beam spot was imaged for a range of lateral positions determined by moving the TS (which are correlated with the angle of incidence from Eq. (5)).

The FWHM of the beam profile (along the $x$ and $y$ directions) for each angle of incidence is represented in Fig. 4. As expected, the minimum spot size is achieved at normal incidence $\left(\theta=0^{\circ}\right)$. At the maximum measured angle of incidence $\theta_{\max }=4.5^{\circ}$, the FWHM of the beam profile in the $x$ direction increased by approximately twice in the direction of stage movement. In the $y$ direction, the variations of the FHWM of the beam profile can be considered as insignificant over the measured angular range.

\section{Validation}

In order to validate our speckle reduction method, we assessed its performance on a scattering, homogeneous sample made of titanium dioxide particles suspended in epoxy resin. The diameter of these particles did not exceed $1 \mu \mathrm{m}$. In particular, we evaluated the impact of the acquisition parameters (namely, the angle of incidence range $\Delta \theta$ and 
Direct en-face, speckle-reduced images using angular-compounded Master-Slave OCT 8

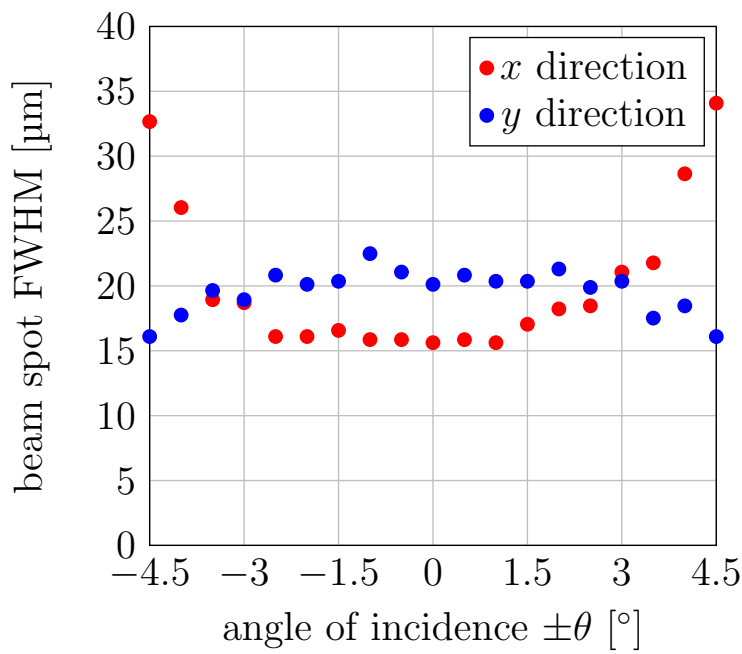

Fig. 4: FWHM of the beam spot versus angle of incidence $\theta$ measured along both the $x$ and $y$ directions.

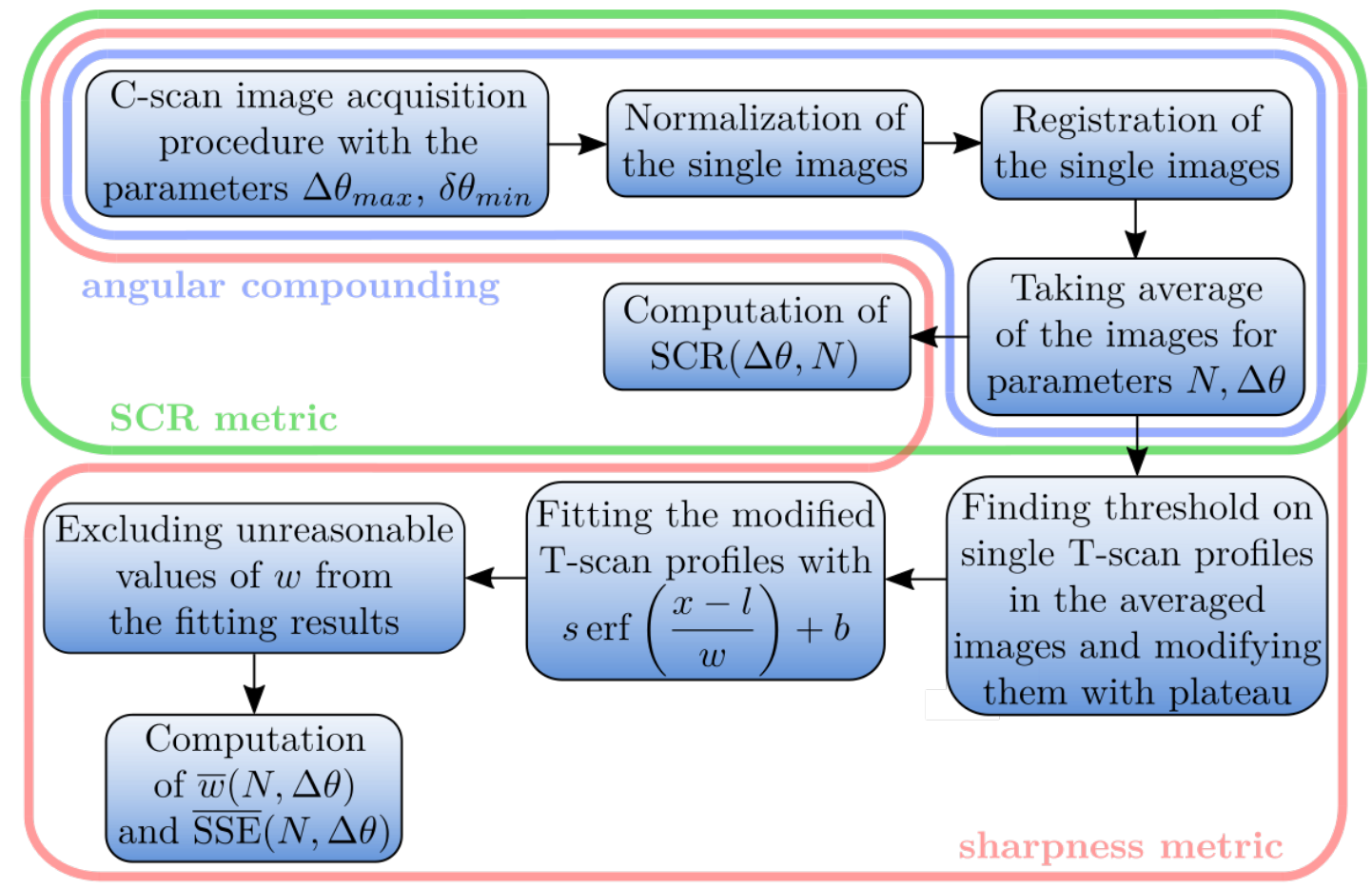

Fig. 5: Diagram of the processes carried out (blue - angular compounding, green - SCR metric, pink/orange - sharpness metric). T-scan stands for a transversal scan profile, and erf is the error function. Other variables are defined in Sec. 3.2.

the number of averaged frames $N$ ) on the speckle contrast ratio of the speckle-reduced image. These results are presented in detail in Section 3.1.

To determine the effect of the aberrations induced by off-axis operation, as discussed 
Direct en-face, speckle-reduced images using angular-compounded Master-Slave OCT 9

in Section 2.3, we also assessed the impact that the angular compounding procedure has on the sharpness of the final speckle-reduced image. This was performed using a scattering line pair phantom printed on card and involved analysing the edge response function for different combinations of $\Delta \theta$ and $N$ values, by modelling an error function on the transverse scan (T-scan) profile taken over a vertically oriented line transition (within the en-face frame). For this purpose, we devised a sharpness metric (Figure 5, red-bounded region) which is described in detail in Section 3.2.

\subsection{SCR quantification metric and its dependence on $\Delta \theta$ and $N$}

The procedure for computing the SCR metric is schematically represented in the diagram in Fig. 5 (blue-bounded region). To compute the speckle-reduced images, stacks of en-face images are acquired for individual angles of incidence (each image being produced by the CMS algorithm using a specific mask, for a selected depth, and represented as an intensity map, with the phase information being discarded), within a range of $\Delta \theta \in\left[-\theta_{\max },+\theta_{\max }\right]$, stepped at an equidistant angular step between images of $\delta \theta_{\min }$, and with the centre of the interval always corresponding to the case where $\theta=0^{\circ}$. Each frame within the stack is then normalised to have the same average intensity.

As the angle of incidence is changed, the imaging plane is modified, and lateral shifts are observed in the en-face OCT images. Prior to image averaging, these enface images need to be registered and aligned. Due to the lack of physical contours in the titanium dioxide sample, the image registration and alignment was carried out by means of a custom MATLAB script based on a normalised cross-correlation between adjacent frames, followed by assessing the average pixel shift between them. If no such registration procedure is performed, the amount of speckle reduction is actually higher than one can reach by pure angular compounding, since we are effectively carrying out spatial averaging. However, to obtain meaningful images and preserve the sharpness of the physical structures, the registration procedure is indispensable; we have employed it in both validation studies using the sharpness metric (Section 3.2) as well as when imaging a biological sample (Section 4), albeit using an intensity-based registration method in the latter case.

To produce a speckle-reduced image, the mean of $N$ frames is then calculated. The theoretical SCR reduction is then given by the expression in Eq. (3). This was performed for different values of the angular range $\Delta \theta$, which for a total of $N$ frames resulted in an angular step of $\delta \theta$ between them, as given by Eq. (6). The speckle contrast ratio (SCR) was calculated using Eq. (2). During SCR computation it was found useful to take the largest possible area $A$ in order to obtain sufficiently representative speckle statistics.

Results both with and without registration are shown in Fig. 6. Note the slightly higher SCR values for the registered case versus the unregistered case, due to the extra spatial averaging caused by lateral shifts of features in the images. For larger $\Delta \theta$ values, these differences vanish and the SCR metric approaches the theoretical model from Eq. (3). This implies complete decorrelation between speckle patterns at this point. 
Direct en-face, speckle-reduced images using angular-compounded Master-Slave OCT10

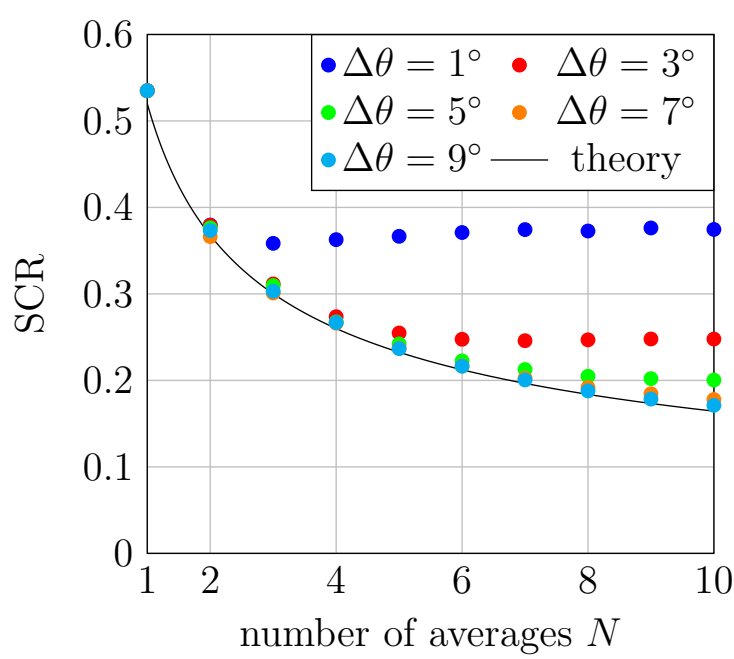

(a) Unregistered images

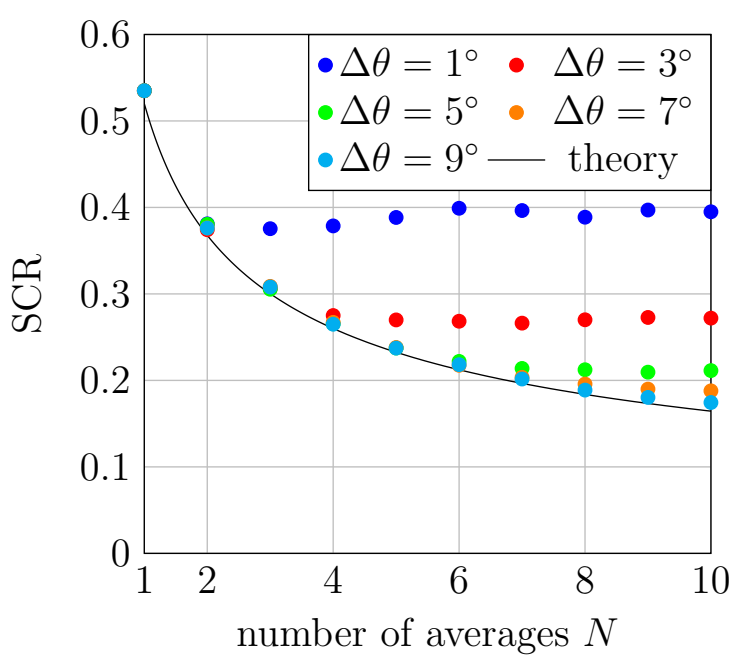

(b) Registered images

Fig. 6: En-face SCR reduction for both unregistered and registered images. Theoretical curves assume fully decorrelated frames.

The main reason of somewhat lower SCR reduction for smaller $\Delta \theta$ is due to residual correlation between speckle patterns. Fig. 7 shows the degree of average correlation of registered frames versus the angular step $\delta \theta$. From this plot it is obvious that for an interframe correlation under $10 \%$ the angular step should be approximately $\delta \theta_{\text {uncorr }} \gtrsim 0.8^{\circ}$. By meeting this condition, one can expect to obtain the highest possible SCR reduction, agreeing with the theoretical model from Eq. (3).

Due to the angular compounding method employed in this study, in which the angle of incidence is varied, we considered the possibility that the speckle statistics might not be constant over the angles of incidence and the imaging depths considered. To explore that possibility, the SCR and the average speckle size for individual frames were calculated over the angle of incidence $\theta$ and relative depth $z-z_{0}$ (with respect to the approximate depth position of the sample surface, $z_{0}$ ).

In order to obtain a value for the average speckle size, we followed the procedure described in Ref. [28]. By taking the auto-correlation of an image (as shown in the plot in Fig. 8), it is possible to measure the mean speckle grain radius, $s$, as the pixel shift value where the correlation value drops below $1 / \mathrm{e}$. This pixel shift value corresponds to a radius of roughly $5 \mu \mathrm{m}$ laterally, or a speckle grain size of $10 \mu \mathrm{m}$.

We have repeated this process for different en-face frames, acquired at different angles of incidence, and also at different imaging depths below the sample surface $z-z_{0}$, with the speckle size (taken at $1 / \mathrm{e}$ ) being constant over the whole range of angles and imaging depths considered, as shown in Figs. 9(a) and 9(b), respectively. In both cases, the SCR for individual frames is also consistent, as shown in Figs. 9(c) and 9(d).

As mentioned in Sec. 2.2 , the $1 / \mathrm{e}^{2}$ spot size $w_{0}$ is dictated by the NA of the imaging system, obtaining $w_{0} \approx 9.6 \mu \mathrm{m}$. This value, when considering the $1 / \mathrm{e}$ width, $\frac{w_{0}}{\sqrt{2}} \approx 6 \mu \mathrm{m}$, 
Direct en-face, speckle-reduced images using angular-compounded Master-Slave OCT11

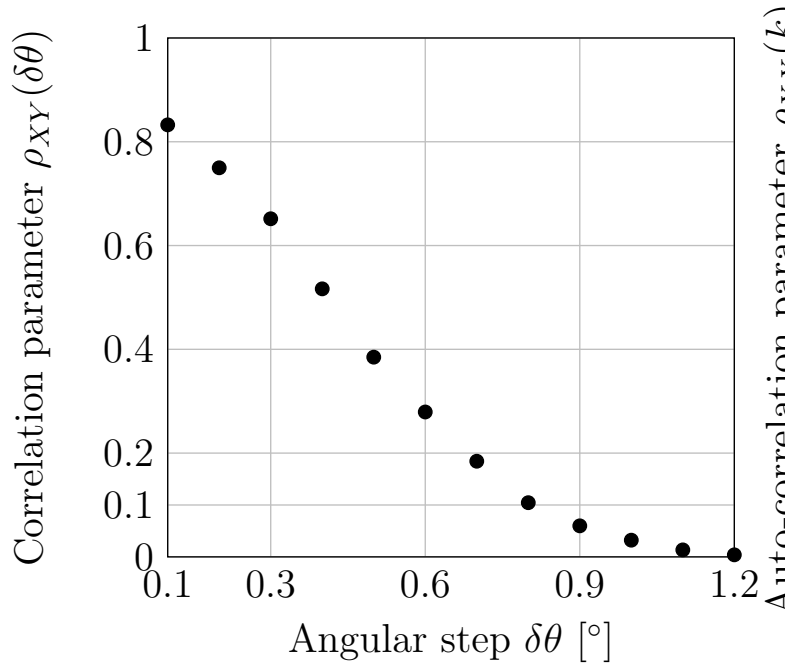

Fig. 7: Correlation $\rho_{X Y}(\delta \theta)$ between two frames versus the angular step $\delta \theta$ between them.

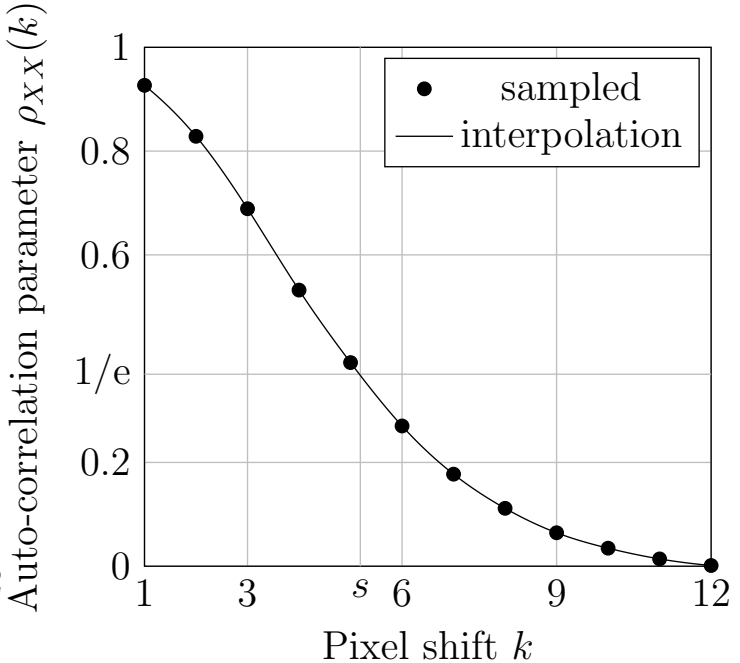

Fig. 8: Auto-correlation $\rho_{X X}(k)$ for a single frame versus pixel shift $k$.

seems to agree with the measured 1/e speckle sizes plotted in Figs. 9(a) and 9(b). Given the large DoF of our imaging system $(\approx 0.7 \mathrm{~mm})$, it is not expected that the speckle size and the SCR would vary with defocusing. The axial range of the system used in this study is dominated by the limited spectrometer resolution, as shown by the plot in Fig. 2 (where a $-6 \mathrm{~dB}$ drop in sensitivity is obtained at $z=1.25 \mathrm{~mm}$ ). 
Direct en-face, speckle-reduced images using angular-compounded Master-Slave OCT12

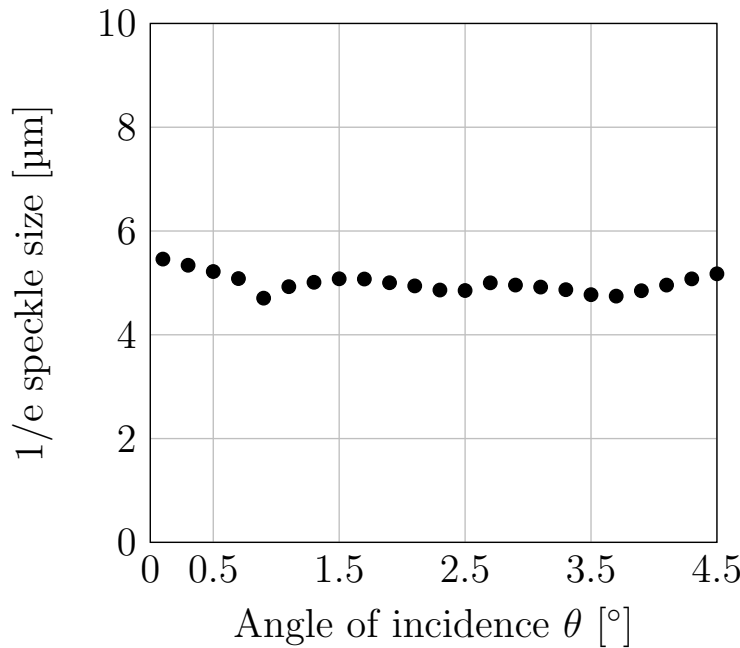

(a) Average speckle size versus angle of incidence $\theta$.

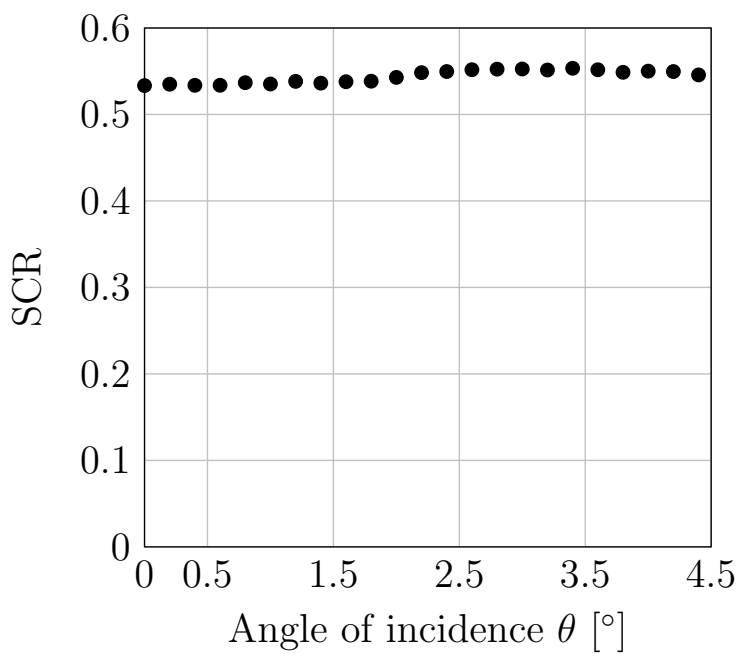

(c) SCR of a single frame versus angle of incidence $\theta$.

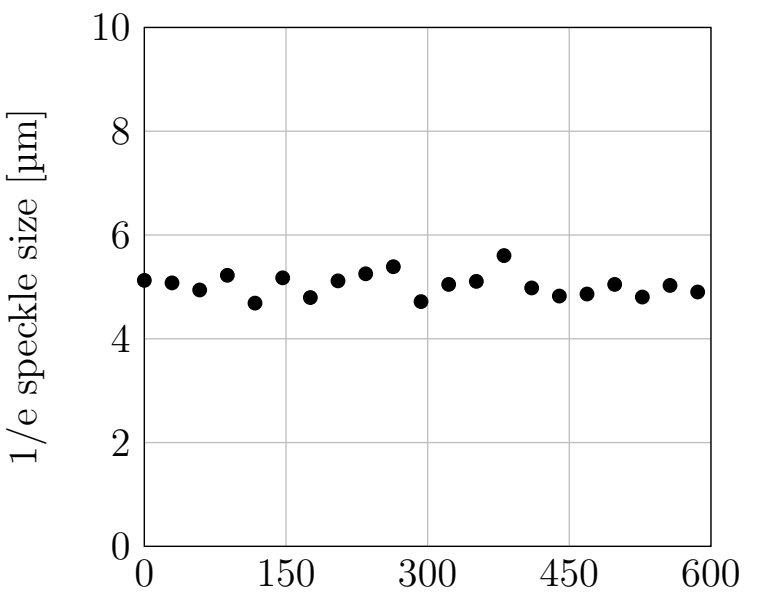

Depth position below surface $z-z_{0}[\mu \mathrm{m}]$

(b) Average speckle size versus relative depth $\Delta z$.

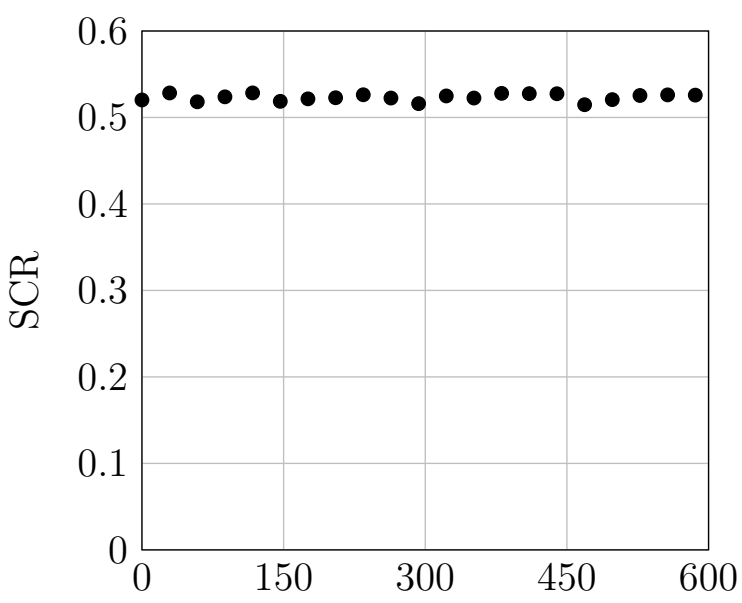

Depth position below surface $z-z_{0}[\mu \mathrm{m}]$

(d) SCR of a single frame versus relative depth $\Delta z$.

Fig. 9: Speckle metric for the titanium dioxide resin sample for single frames. There is no dependency of SCR and speckle size (taken at 1/e of the auto-correlation function) either on the angle of incidence $\theta$ or the relative depth position below the surface, $z-z_{0}$. 


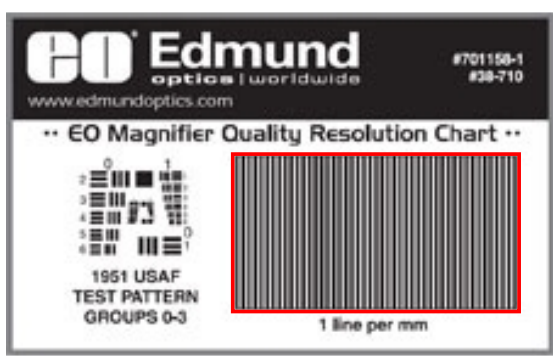

Fig. 10: EO resolution chart (red rectangle) used for sharpness measurements.

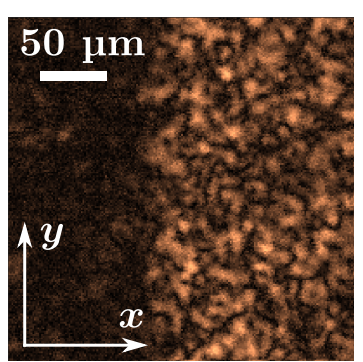

Fig. 11: Example en-face image showing edge transition between a dark and light line. The black, low-signal region on the left corresponds to the dark line, due to the attenuation of the ink, while the lighter region on the right corresponds to the white card background between the lines, which exhibits speckles.

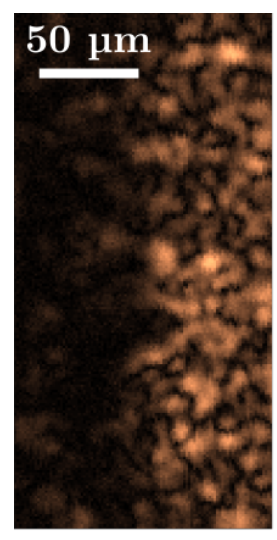

original

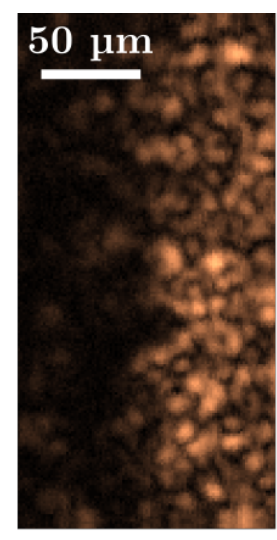

$$
\begin{gathered}
N=2 \\
\Delta \theta=1^{\circ}
\end{gathered}
$$

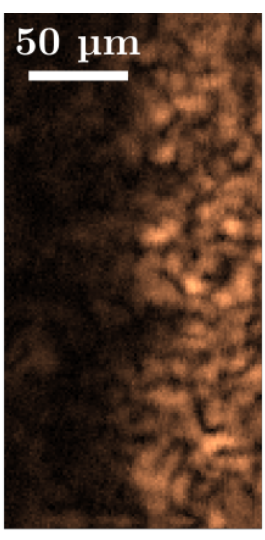

$$
\begin{aligned}
N & =2 \\
\Delta \theta & =9^{\circ}
\end{aligned}
$$

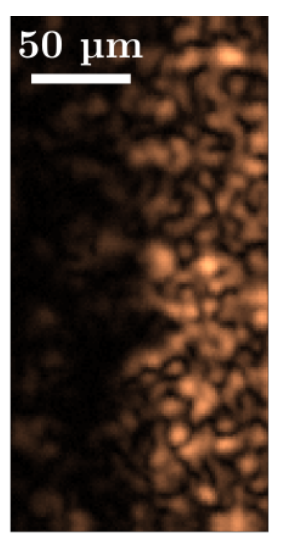

$$
\begin{gathered}
N=10 \\
\Delta \theta=1^{\circ}
\end{gathered}
$$

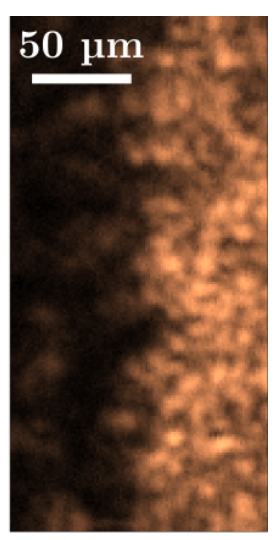

$$
\begin{gathered}
N=10 \\
\Delta \theta=9^{\circ}
\end{gathered}
$$

Fig. 12: Examples of the edge transition after speckle reduction for various representative angular compounding parameters $N$ and $\Delta \theta$.

The impact of speckle pattern averaging on the image sharpness was investigated using a Ronchi line pattern embedded in a vinyl laminate Magnifier Quality Resolution Chart (Fig. 10). A sharpness metric was established by analysing the transition between a light and a dark line within the pattern (Fig. 11). Examples of the edge transition for several combinations of $N$ and $\Delta \theta$ values are shown in Fig. 12.

The first four steps of the procedure for evaluating the sharpness metric are shared with those employed in the evaluation of the SCR metric (Fig. 5). After the speckle- 
Direct en-face, speckle-reduced images using angular-compounded Master-Slave OCT14

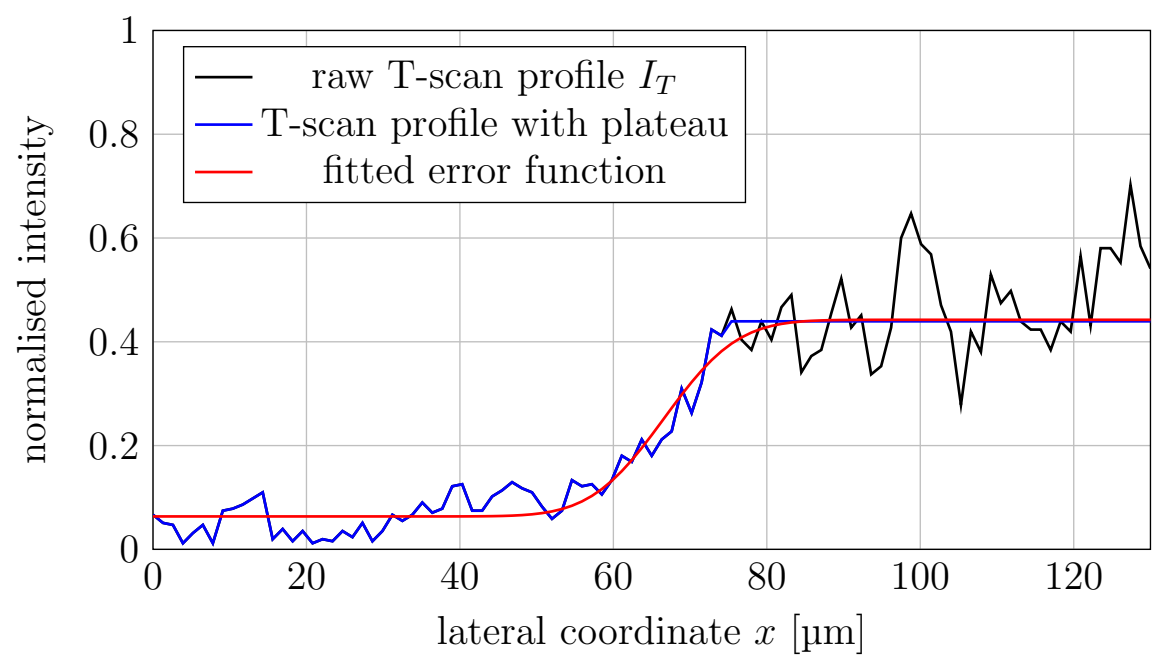

Fig. 13: An example of the fitting procedure being applied to a single T-scan profile (black), its plateaued modification (blue) and fitted error function (red).

reduced image is obtained, T-scan intensity profiles $I_{T}$ along the $x$ direction of the frame (Fig. 11) are extracted for all $y$ positions in the frame. An example of one such T-scan (transversal scan) profile $I_{T}$ is shown as the black trace in Fig. 13.

These T-scans are performed over a transition from a light to a dark region within the Ronchi pattern are then used to estimate the sharpness of the speckle-reduced image. To this goal, the scaled error function $s$ erf $\left(\frac{x-l}{w}\right)+b$, where $x$ is a coordinate along the Tscan profile and $s, l, w$ and $b$ are the scale, shift, width and bias parameters of the error function, respectively. This function is fitted to the data [29] and its fitted parameter $w$ is extracted. This describes the width of the transition (a measure of the image sharpness), whilst the summed squared error (SSE) of the fitting is used to describe the 'smoothness' of the transition (effectively another measure of speckle reduction).

Before carrying out the fitting operation, each T-scan profile was modified to present a flat plateau in the higher intensity side of the profile (covering the paper background in the card), by setting $I_{T}\left(x \geq x_{\bar{I}_{T, p l a t}}\right)=\bar{I}_{T, p l a t}$, where $x_{\bar{I}_{T, p l a t}}$ is the pixel $x$ coordinate for which the intensity profile $I_{T}$ first reaches the mean value over the T-scan region with speckles, $\bar{I}_{T, p l a t}$. An example of a modified profile with the flat plateau is shown in Fig. 13 as the blue trace. Having this modified profile is important as the residual speckles in the upper plateau will otherwise affect the fitting operation, while we are only interested in the transition, and not on the speckles which lie beyond it.

Following the error function fitting operation over all the individual T-scans (an example of which is shown as the blue trace in Fig. 13), we have taken the mean of all fitted $w$ values, $\bar{w}(N, \Delta \theta)$, which describes the average 'slope' of the transition (its blur). It was necessary to exclude some unreasonable values of $w$, (which would otherwise skew the mean) and include only those values which lie in the interval $[0.5,50] \mathrm{px}$. Our reasoning is that, for $w<0.5 \mathrm{px}$ with the sampling rate $1 / \Delta x=1 \mathrm{px}^{-1}$, the 
Direct en-face, speckle-reduced images using angular-compounded Master-Slave OCT15

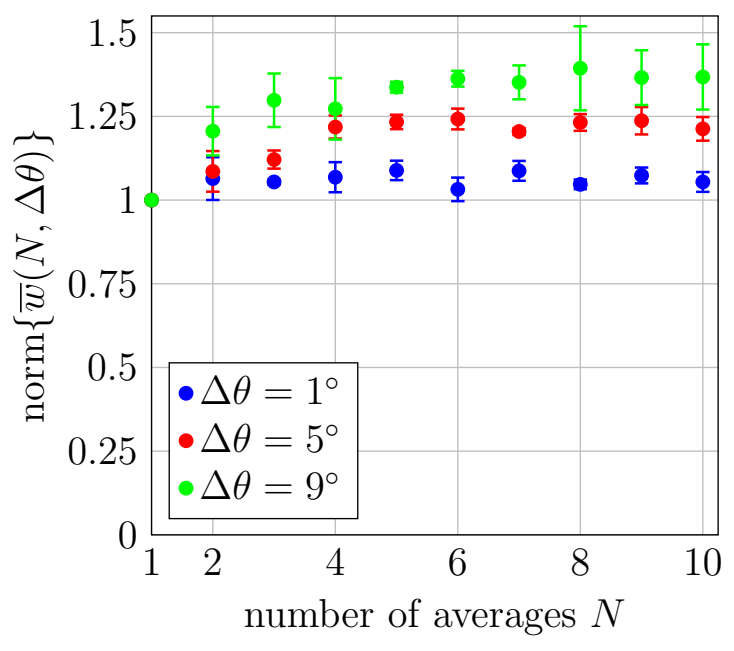

(a)

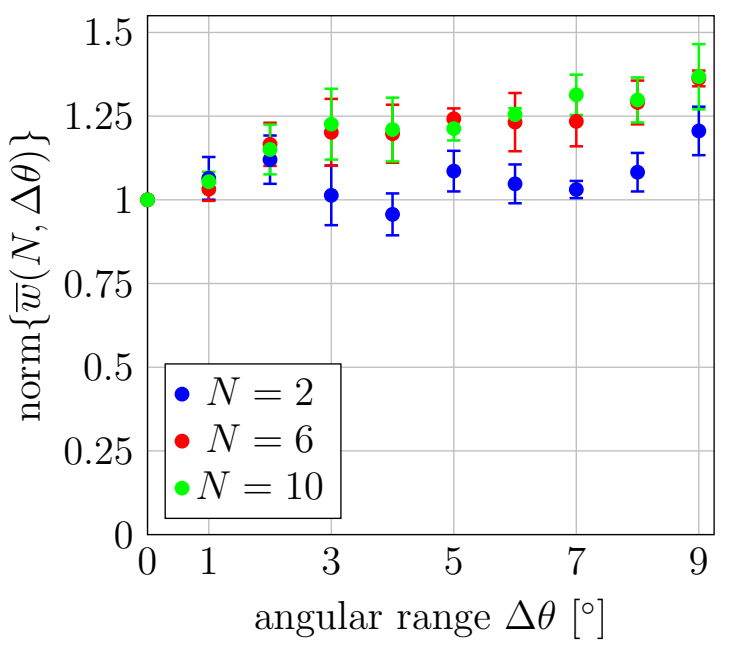

(b)

Fig. 14: Distribution of the normalised mean of the fitted transition width $\bar{w}(N, \Delta \theta)$ between two lines of the Ronchi pattern, for varying number of averages $N$ (a) and angular ranges $\Delta \theta$ (b). Values larger than 1 mean wider, thicker transitions than those measured for when no angular compounding is used.

error function becomes under-sampled; furthermore, we do not expect the transition to be wider than 50 px if the length of the measured T-scan profiles in Fig. 12 is 100 px. Therefore, the values excluded refer to T-scan profiles where the error function fit yielded erroneous values. Figures 14(a) and 14(b) describe the dependencies of $\bar{w}$ on both the number of averages $N$ and the angular range $\Delta \theta$ employed in the speckle reduction procedure. To clarify these results further, parameters $\bar{w}(N, \Delta \theta)$ were normalised to the case of no speckle reduction (no angular compounding) as

$$
\operatorname{norm}\{\bar{w}(N, \Delta \theta)\} \equiv \frac{\bar{w}(N, \Delta \theta)}{\bar{w}\left(1,0^{\circ}\right)},
$$

with $\bar{w}\left(1,0^{\circ}\right)$ corresponding to the case where no angular compounding (speckle reduction) was carried out. From Figs. 14(a) and 14(b) we can see that values of $\operatorname{norm}\{\bar{w}(N, \Delta \theta)\}$ over $N$ and $\Delta \theta$ are mildly increasing and bigger than 1 , which, with respect to Eq. (7), means that the transition is becoming wider with increasing $N$ or $\Delta \theta$. In other words, the angular compounding introduces a slight blur to the transition. Some examples of how the transition is affected after angular compounding (averaging) for some parameters $N$ and $\Delta \theta$ are shown in Fig. 12.

After averaging of images to achieve speckle reduction, a 'smoothness' of the transition intensity profile can be assessed by the SSE parameter, computed from the fitting error function calculated during the previous fitting procedure (but without the flat plateau modification mentioned above). The summed squared error (SSE) expresses the deviation from the fitted function and it can be used as a measure of variation (dispersion) of data. We have taken the mean of all $\operatorname{SSE}$ values, $\overline{\operatorname{SSE}}(N, \Delta \theta)$. Again, 
on the mean calculation we excluded those values of SSEs for which the corresponding fitted $w$ value did not lie in the interval $[0.5,50]$. In Figs. 15(a) and 15(b), $\overline{\operatorname{SSE}}(N, \Delta \theta)$ for a given en-face frame is plotted against the number of averages $N$ and the angular ranges $\Delta \theta$, where the results were normalised as

$$
\operatorname{norm}\{\overline{\operatorname{SSE}}(N, \Delta \theta)\} \equiv \frac{\overline{\operatorname{SSE}}(N, \Delta \theta)}{\overline{\operatorname{SSE}}\left(1,0^{\circ}\right)},
$$

with $\overline{\operatorname{SSE}}\left(1,0^{\circ}\right)$ corresponding to the case where no angular compounding (speckle reduction) was carried out. Following Eq. (8), values of the $\operatorname{norm}\{\operatorname{SSE}(N, \Delta \theta)\}$ lower than 1 mean a reduction in the intensity fluctuations along the T-scan, and it is evident from the plots in Figs. 15(a) and 15(b) that with increasing $N$ or $\Delta \theta$ one can attain lower variations of the pixel values along the direction of each T-scan in the image. These results are consistent with the SCR metric in Section 3.1, where the SCR is computed as a normalised standard deviation of the intensity, as shown in Eq. (2).

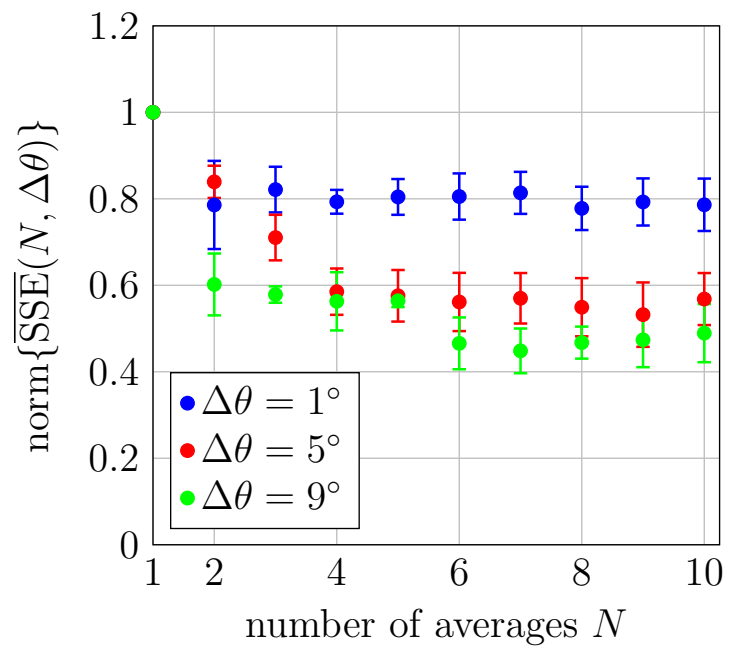

(a)

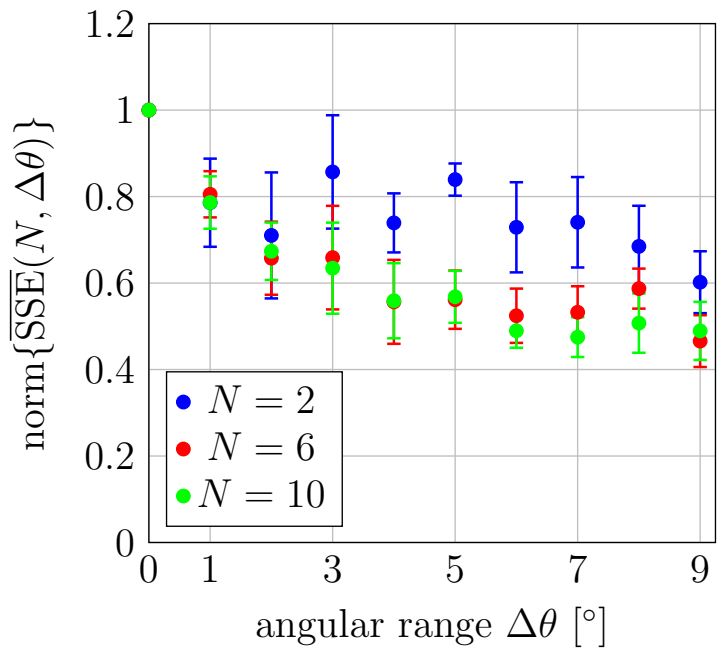

(b)

Fig. 15: Distribution of the normalised SSE metric calculated for the T-scan intensity profiles $\overline{\operatorname{SSE}}(N, \Delta \theta)$ straddling over two lines of the Ronchi pattern, for varying number of averages $N$ (a) and varying angular ranges $\Delta \theta$ (b). Values lower than 1 mean better agreement of data with the fit than observed for no angular compounding.

\section{Biological sample imaging}

We further tested the en face speckle reduction method by imaging a biological sample, in the form of freshly cut potato cells. The procedure for generating angular compounded images was the same as before (the angular compounding step in the block diagram in Fig. 5). Now, for the image registration, the intensity-based MATLAB imregister function was utilised, since the samples considered here are heterogeneous 
in structure. Figs. 16 and 17 show the speckle-reduced en-face images of the sample for increasing number of averages $N$ and increasing angular range $\Delta \theta$, respectively.

For a fixed angular range $\Delta \theta=4^{\circ}$, increasing the number of averages $N$ (Fig. 16) tends to improve the speckle reduction, increasing the visibility of the cell structures, and removing spurious details. Most of the improvement occurs after a small number of averages, and there is little further benefit observable beyond $N=10$. This is in agreement with the speckle contrast ratio measurement reported earlier.

Conversely, for a fixed $N=10$, a larger angular range $\Delta \theta$ (Fig. 17) results in a lower speckle contrast visibility, while the blur in cell structures increases. This is likely in part due to a slight tilting of the raw B-scans for larger angles of incidence. The en-face images from each cube are then slightly out of plane with each other. Since the sample structure varies in depth, this then leads to blurring of features along both both lateral and depth directions.

In addition, increased geometrical aberrations are present at larger $\Delta \theta$ values (as discussed in Section 2.3). Therefore, a trade-off is necessary between the degree of speckle reduction and the sharpness of structures.

Since the sample employed in the validation analysis carried out in Section 3 was uniform in depth, no such trade-off was necessary.

\section{Discussion and Conclusions}

In this report, we have demonstrated speckle reduction in en-face optical coherence tomography images by means of angular compounding using a custom-designed probe head. These OCT images were obtained by Complex Master-Slave (CMS) OCT processing, in conjunction with a spectrometer-based OCT system operating at 840 $\mathrm{nm}$, with an axial resolution of $6.2 \mu \mathrm{m}$ and a lateral resolution of $10 \mu \mathrm{m}$ (at normal incidence). The CMS-OCT processing allows direct rendering of en-face images. This is highly advantageous in terms of time required by a repetitive process such as the one employed here, which requires several data acquisition steps, for several angular orientations of the probe head. Therefore the processing time necessary to display a speckle-reduced en-face image is reduced in comparison with conventional FFT-based processing. This is due to the CMS-OCT method removing the need to reconstruct the entire volume before extracting the en-face images, unlike conventional FFT-based OCT processing. It also removes the need to perform other operations on each channelled spectrum acquired such as $k$-domain linearisation and dispersion compensation.

This study has evaluated the impact of different combinations of the two free parameters affecting the angular compounding method (the number of acquired frames $N$ and the range of angles of incidence $\Delta \theta$ ) on the efficacy of speckle reduction. Specifically, the image sharpness in the transition between two lines of a Ronchi line pattern was analysed.

To assess the efficacy of the speckle removal, we used the speckle contrast ratio (SCR) metric as described previously in the literature [24, 25, 26, 2]. All of these tests 
Direct en-face, speckle-reduced images using angular-compounded Master-Slave OCT18

(a) original

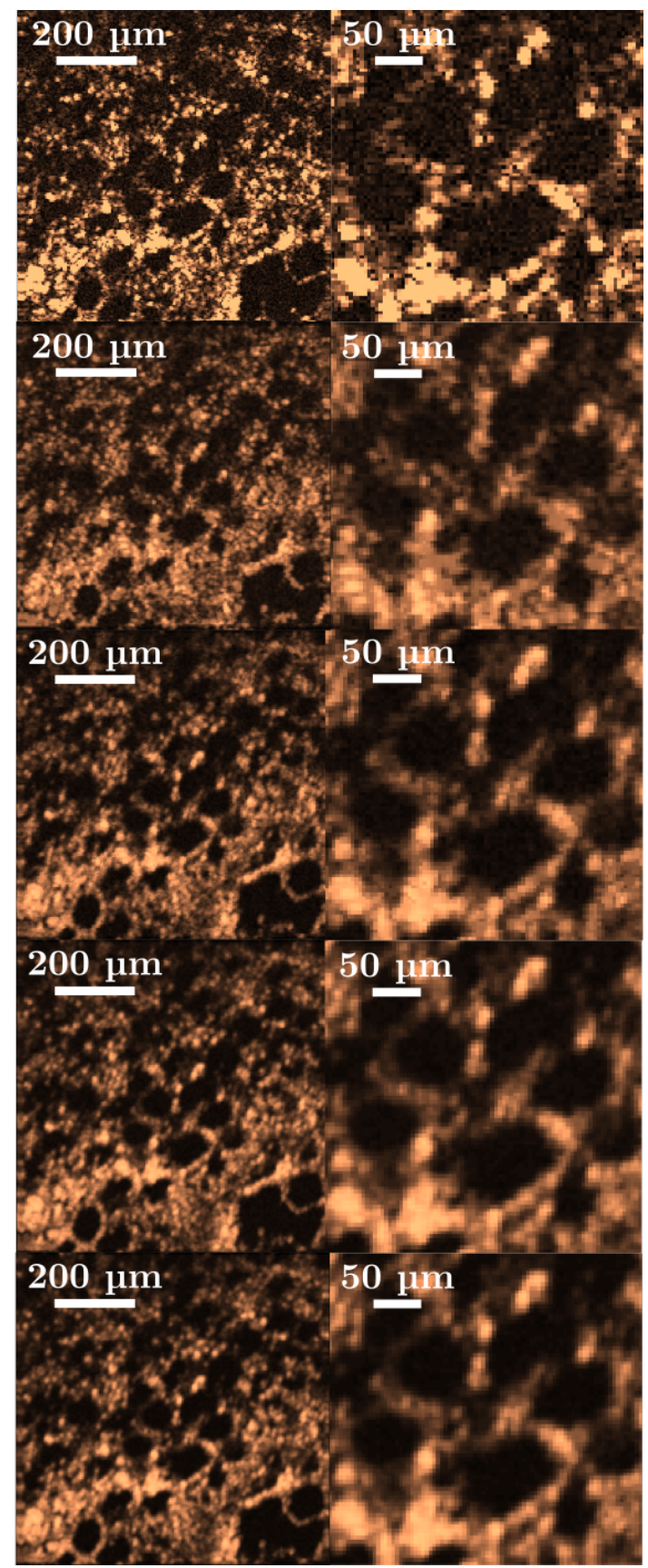

Fig. 16: Angular compounding for en-face images of a potato sample. Angular range is fixed to the value $\Delta \theta=4^{\circ}$ and the number of averages $N$ increases from the top to the bottom row. The images in the right column show a zoom on a part of the en-face frame, centred on a group of cells. With increasing number of averages, the visibility of the cell structures improves due to speckle contrast reduction. 
Direct en-face, speckle-reduced images using angular-compounded Master-Slave OCT19

(a) original

(b) $\Delta \theta=2^{\circ}$

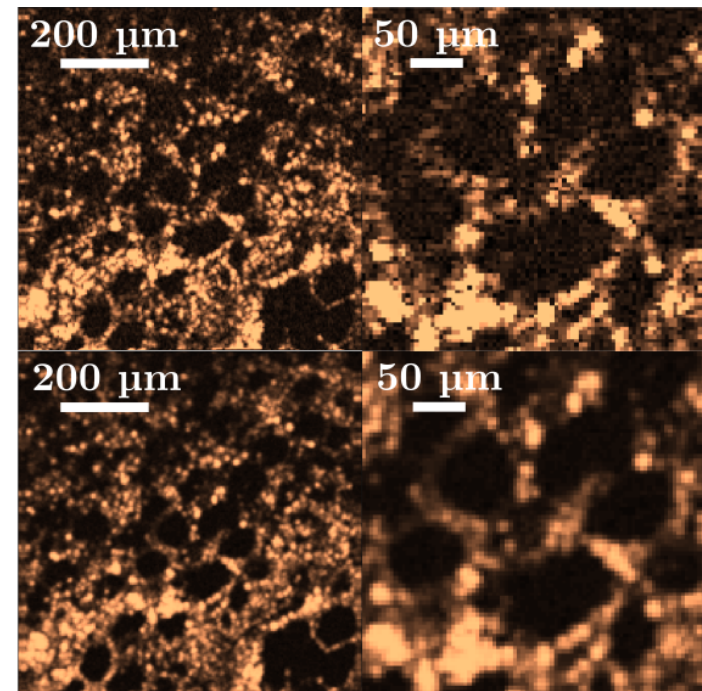

(c) $\Delta \theta=4^{\circ}$

(d) $\Delta \theta=6^{\circ}$

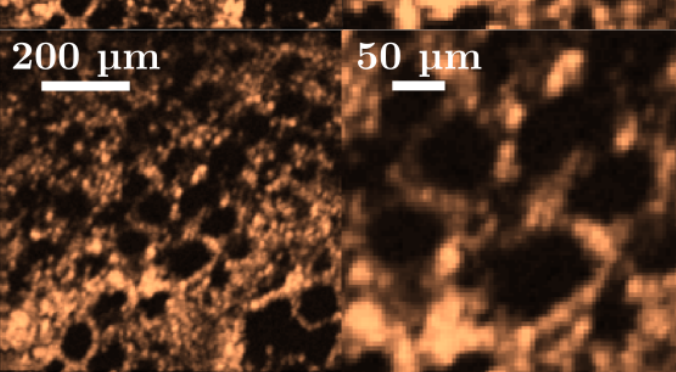

(e) $\Delta \theta=8^{\circ}$

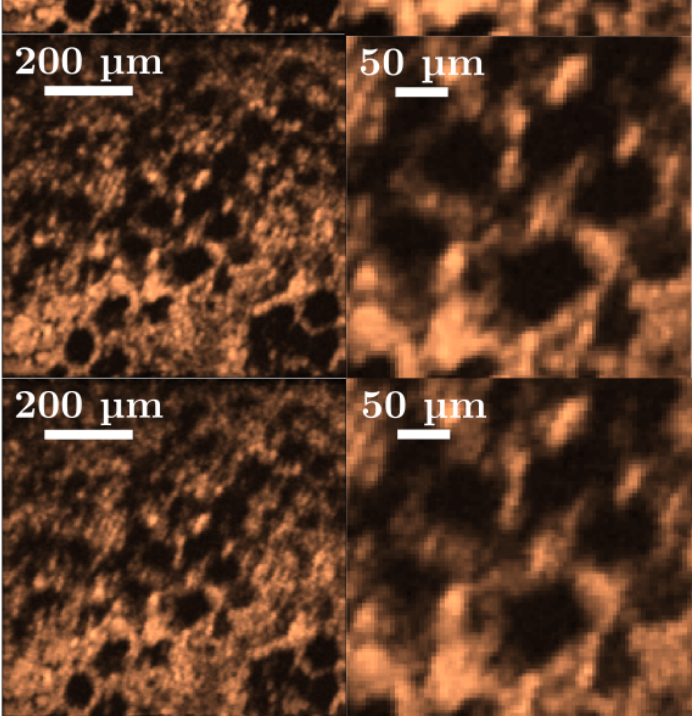

Fig. 17: Angular compounding for en-face images of a potato sample. Number of averages is fixed as $N=10$ and angular range $\Delta \theta$ increases from the top to bottom row. The images in the right column show a zoom on a part of the en-face frame, centred on a group of cells. With increased angular range, speckle contrast diminishes, but the sharpness of features also deteriorates, with the cell walls becoming more blurred due to out-of-plane averaging. 
were carried out on an homogeneous phantom, which yielded the same SCR and speckle size for the range of imaging depths and angles of incidence studied. It was found that the speckle in the images become progressively reduced for an increasing number of acquired frames $N$. However, after a certain number of averages the improvement plateaus and there is little or no further benefit to additional averages. This is because, as $N$ increases for a given total angular range, the step between each image decreases. Eventually, a limit is reached where the speckle patterns are no longer fully de-correlated between images.

For a fixed number of acquired frames, a higher degree of speckle reduction can be achieved with a larger angular range $\Delta \theta$, although at extreme angles, the quality of the imaging beam is degraded significantly by aberrations such as coma. It is also important to ensure that the frames being averaged are properly registered in order to avoid loss of resolution. While our initial analysis with unregistered images actually produced better speckle reduction (i.e. lower SCR), this was due to averaging different regions across images, and hence significant image blurring was observed.

We also studied the impact on image quality caused by the angular compounding method. Due to the specular nature of reflections from resolution phantoms such as USAF glass targets, it was difficult to assess the imaging resolution for varying $\Delta \theta$ due to limited light collection at non-normal angles of incidence. Moreover, due to the thickness of the glass phantom, additional artefacts appeared on the image which masked the true imaging resolution. Observing the imaging beam output with a 2D camera allowed the spot size to be measured; while this does not provide a direct measurement of the imaging resolution, it did reveal the optical aberrations (primarily coma) introduced by the beam walk-off needed for the non-normal angles of incidence.

A sharpness metric for the imaging process was devised with a $1 \mathrm{lp} / \mathrm{mm}$ Ronchi pattern printed on an imaging resolution card. Due to the scattering nature of the target, good light collection was obtained throughout the whole $\Delta \theta$ range. We could then quantify the step transition between a light and a dark line across each en-face frame (for different sets of $N$ and $\Delta \theta$ ) by fitting an error function to each T-scan in the frame. Increases in $N$ and $\Delta \theta$ both lead to a broadening of the transition, although plateaus were observed for constant $\Delta \theta$ and increasing $N$, which seems to correlate with the decrease in beam quality observed for increasing $\Delta \theta$. We have also plotted the standard error of the function fit, which followed the trends already found for the SCR for increasing $N$. This is to be expected, as the speckle reduction procedure effectively smooths the T-scans, bringing their profiles closer to that of the fitted erf model and therefore lowering the fitting error. This can therefore be thought of an additional quantification of the degree of speckle reduction.

The biological sample images mirror the findings presented in the "Validation" section, with one additional point worth noting - both phantoms used are relatively uniform in depth, whereas the potato sample employed here is not. Since the variation of the angle of incidence also introduces a depth-dependent tilt in the images, it is possible to have some out-of-plane components in some of the images, especially those 
Direct en-face, speckle-reduced images using angular-compounded Master-Slave OCT21

acquired at more extreme angles. This is particularly evident when using $\Delta \theta$ values larger than $6^{\circ}$, as shown in Fig. 17.

Taking into consideration the findings for the imaging system used here, an intermediate angular range of $\Delta \theta \sim 3-5^{\circ}$ is shown to significantly reduce speckle. Moreover, after 4-6 acquired frames (within that range of angles) the SCR values have reached a plateau and it is therefore unnecessary to acquire any further frames. The overall acquisition time can therefore be kept within a reasonable level. With this choice of parameters, some degree of blurring can still be expected due to a decrease in the imaging beam quality and any imperfections in the image registration prior to averaging.

Even with an angular range of less than $5^{\circ}$, a non-negligible tilt occurs in the images acquired at larger angles of incidence. This introduces some loss of resolution in the speckle reduced image. If reconstructing entire volumes before performing an en-face cut (as in conventional FFT-based processing) this could be fixed by a registration procedure which allows for B-scan tilt. However, this is not option when extracting en-face slices at high-speed using the CMS-OCT technique.

However, as the tilt is simply a fixed geometrical effect which could be measured in advance, it would be possible to customise the CMS-OCT processing procedure to correct the tilt. Rather than generating a single CMS-OCT mask to extract points at the same depth for each pixel in the en-face image, a custom set of masks could be pregenerated for each angle, designed to extract an oblique plane which would counteract the tilt. This would then directly correct the angle-dependent tilt without the need to reconstruct an entire volume and tilt each B-scan.

The results presented here complement the method of angular compounding for speckle reduction. CMS-OCT processing opens up a faster approach to averaging enface images which are directly extracted from the raw unprocessed set of spectra at higher speed, helping make speckle reduction in en-face imaging a realistic prospect for applications which require real-time imaging.

\section{Acknowledgements}

Erasmus+ programme (RR); Engineering and Physical Sciences Research Council (EPSRC), "REBOT" project, EP/N019318/1 (MJM/MRH/AB/AP); Biotechnology and Biological Sciences Research Council (BBSRC), "5DHiResE" project, BB/S016643/1 (MJM/AB/AP); National Institute for Health Research Biomedical Research Centre at Moorfields Eye Hospital NHS Foundation Trust (NIHR) (AP); the UCL Institute of Ophthalmology, University College London (AP); Royal Society Wolfson research merit award (AP).

\section{References}

[1] J Fujimoto and W Drexler. Introduction to optical coherence tomography. In Optical coherence tomography, pages 1-45. Springer, 2008. 
Direct en-face, speckle-reduced images using angular-compounded Master-Slave OCT22

[2] M Hughes, M Spring, and A Podoleanu. Speckle noise reduction in optical coherence tomography of paint layers. Applied Optics, 49(1):99, January 2010.

[3] J M Schmitt, S H Xiang, and K M Yung. Speckle in optical coherence tomography. J. Biomed. Opt., 4(1):95-105, January 1999.

[4] J Goodman. Speckle Phenomena in Optics: Theory and Applications. 012007.

[5] J M Schmitt. Array detection for speckle reduction in optical coherence microscopy. Phys. Med. Biol., 42(7):1427-1439, July 1997.

[6] J W Goodman. Some fundamental properties of speckle*. JOSA, 66(11):1145-1150, November 1976.

[7] F Forsberg, S Leeman, and J A Jensen. Assessment of hybrid speckle reduction algorithms. Physics in Medicine and Biology, 36(11):1539-1549, November 1991.

[8] N Iftimia, B E Bouma, and G J Tearney. Speckle reduction in optical coherence tomography by "path length encoded" angular compounding. JBO, 8(2):260-264, April 2003.

[9] A E Desjardins, B J Vakoc, G J Tearney, and B E Bouma. Speckle reduction in OCT using massively-parallel detection and frequency-domain ranging. Opt. Express, OE, 14(11):47364745, May 2006.

[10] A E Desjardins, B J Vakoc, W Y Oh, S M R Motaghian Nezam, G J Tearney, and B E Bouma. Angle-resolved Optical Coherence Tomography with sequential angular selectivity for speckle reduction. Opt. Express, OE, 15(10):6200-6209, May 2007.

[11] H Wang and A M Rollins. Speckle reduction in optical coherence tomography using angular compounding by B-scan Doppler-shift encoding. JBO, 14(3):030512, May 2009.

[12] L Duan, H Y Lee, G Lee, M Agrawal, G T Smith, and A K Ellerbee. Single-shot speckle noise reduction by interleaved optical coherence tomography. JBO, 19(12):120501, December 2014.

[13] W Shi, C Chen, J Jivraj, Y Dobashi, W Gao, and V X Yang. 2d MEMS-based high-speed beamshifting technique for speckle noise reduction and flow rate measurement in optical coherence tomography. Opt. Express, 27(9):12551, April 2019.

[14] Y Winetraub, C Wu, G P Collins, S Chu, and A de la Zerda. Upper limit for angular compounding speckle reduction. Appl. Phys. Lett., 114(21):211101, May 2019.

[15] I Rubinoff, L Beckmann, Y Wang, A A Fawzi, X Liu, J Tauber, K Jones, H Ishikawa, J S Schuman, R Kuranov, and H F Zhang. Speckle reduction in visible-light optical coherence tomography using scan modulation. Neurophotonics, 6(4):041107, September 2019.

[16] O Liba, M D Lew, E D SoRelle, R Dutta, D Sen, D M Moshfeghi, S Chu, and A de la Zerda. Speckle-modulating optical coherence tomography in living mice and humans. Nat Commun, 8(1):15845, August 2017.

[17] M Xu, C Tang, M Chen, Y Qiu, and Z Lei. Texture preservation and speckle reduction in optical coherence tomography using the shearlet-based total variation algorithm. Optics and Lasers in Engineering, 122:265-283, November 2019.

[18] C Cuartas-Vélez, R Restrepo, B E Bouma, and N Uribe-Patarroyo. Volumetric non-local-means based speckle reduction for optical coherence tomography. Biomedical Optics Express, 9(7):3354, July 2018.

[19] G Gong, H Zhang, and M Yao. Speckle noise reduction algorithm with total variation regularization in optical coherence tomography. Optics Express, 23(19):24699-24712, September 2015.

[20] A Bradu, S Rivet, and A Podoleanu. Master/slave interferometry - ideal tool for coherence revival swept source optical coherence tomography. Biomedical Optics Express, 7(7):2453, July 2016.

[21] S Rivet, M Maria, A Bradu, T Feuchter, L Leick, and A Podoleanu. Complex master slave interferometry. Optics Express, 24(3):2885, February 2016.

[22] A G Podoleanu and A Bradu. Master-slave interferometry for parallel spectral domain interferometry sensing and versatile 3d optical coherence tomography. Opt. Express, 21(16):19324-19338, Aug 2013.

[23] M J Marques, S Rivet, A Bradu, and A Podoleanu. Novel software package to facilitate operation 
Direct en-face, speckle-reduced images using angular-compounded Master-Slave OCT23

of any spectral (Fourier) OCT system. In S A Boppart, M Wojtkowski, and W Y Oh, editors, Optical Coherence Imaging Techniques and Imaging in Scattering Media II, page 5, Munich, Germany, August 2017. SPIE.

[24] M Bashkansky and J Reintjes. Statistics and reduction of speckle in optical coherence tomography. Opt. Lett., 25(8):545, April 2000.

[25] B Karamata, K Hassler, M Laubscher, and T Lasser. Speckle statistics in optical coherence tomography. Journal of the Optical Society of America A, 22(4):593, April 2005.

[26] M Pircher, E Götzinger, R A Leitgeb, A F Fercher, and C K Hitzenberger. Speckle reduction in optical coherence tomography by frequency compounding. JBO, 8(3):565-570, July 2003.

[27] O Svelto. Principles of lasers. Springer, 4 edition, 2009.

[28] I Hamarová, P Šmíd, P Horváth, and M Hrabovsky. Methods for determination of mean speckle size in simulated speckle pattern. Measurement Science Review, 14:177-182, 062014.

[29] C W Merkle, S P Chong, A M Kho, J Zhu, A Dubra, and V J Srinivasan. Visible light optical coherence microscopy of the brain with isotropic femtoliter resolution in vivo. Opt. Lett., 43(2):198-201, Jan 2018. 